\title{
Frequency mixing at an electromagnetically induced transparency like metasurface loaded with gas as a nonlinear element
}

\author{
Yasuhiro Tamayama ${ }^{1,}$ a) and Takuya Yoshimura ${ }^{1}$ \\ Department of Electrical, Electronics and Information Engineering, Nagaoka University of Technology, \\ 1603-1 Kamitomioka, Nagaoka, Niigata 940-2188, Japan
}

(Dated: 15 November 2018)

Local electromagnetic field enhancement in resonant metamaterials is useful for efficient generation of nonlinear phenomena; however, the field enhancement is suppressed by losses of nonlinear elements in metamaterials. For overcoming this issue, we investigate the nonlinear response of an electromagnetically induced transparency like metasurface loaded with gas as the nonlinear element. To induce nonlinearity in the gas associated with discharges, an electromagnetic wave with a modulated amplitude is incident on the metasurface. The measured waveform and spectrum of the transmitted electromagnetic wave, along with light emission from the discharge microplasma, reveal that frequency mixing can occur on the metasurface. The parameter dependence of the conversion efficiency of the frequency mixing phenomenon shows that the efficiency is determined almost entirely by the ratio of the duration of microplasma generation to the modulation period of the incident wave amplitude. This result implies that the frequency mixing is derived from a binary change in the transmittance of the metasurface caused by the generation and quenching of the microplasma.

There has been considerable interest in enhancing nonlinear phenomena using metamaterials. $\frac{1}{1}$ When an electromagnetic wave is incident on a resonant metamaterial, the electromagnetic energy becomes compressed into a small volume in the metamaterial. If a nonlinear element is excited by the enhanced local electromagnetic field, nonlinear phenomena can be efficiently generated. To date, various nonlinear phenomena, such as frequency conversion,,-18 power-dependent responses,,$\frac{19-25}{2}$ and nonlinear wave propagation $26-31$ have been investigated.

The enhancement factor of the local electromagnetic field in a resonant metamaterial is inversely related with the losses in its unit structure. In the optical region, nonlinear dielectric resonators ${ }^{7-9}$ would be suitable for the unit structure, since nonlinear dielectrics possess small losses. In contrast, lower frequency regions, including microwave and terahertz, require electronic devices, such as varactor diodes, to be introduced as nonlinear elements. 1 These electronic devices have relatively high losses, which suppress the local electromagnetic field enhancement.

In this study, we investigate a metasurface loaded with gas as a nonlinear element to develop a method for preventing the suppression of the resonant enhancement of local electromagnetic fields. Gases have low linear losses, and exhibit discharges under the application of a strong electric field. Thus, we consider gases to be suitable for the efficient generation of nonlinear phenomena in resonant metamaterials, especially at lower frequencies. In addition, discharges cause abrupt changes in the electromagnetic characteristics of gases. Therefore, it may be possible to generate highly nonlinear phenomena in gas-loaded resonant metamaterials. In this paper, we show that frequency mixing can be generated using the

\footnotetext{
a) Electronic mail: tamayama@vos.nagaokaut.ac.jp
}

dynamic changes in the electromagnetic response of a metasurface associated with a gas discharge in its unit structure.

Figure 1(a) shows the metasurface structure used in this study. The details of the electromagnetic response of the metasurface are described in our previous papers, $, 32,33$ and we briefly review them here. This metasurface is composed of two radiatively coupled cut-wire resonators with slightly different resonance frequencies, and exhibits an electromagnetically induced transparency (EIT) ${ }^{34,35}$ -like electromagnetic response 36 The EIT-like transmission peak frequency is $f_{0}=3.031 \mathrm{GHz}$, which is approximately the average of the resonance frequencies of the two cut-wire resonators, $3.016 \mathrm{GHz}$ and $3.059 \mathrm{GHz}$. When an electromagnetic wave with a frequency around $f_{0}$ is incident on the metasurface, a strongly enhanced local electric field is induced at the gaps of the cut-wire resonators. The amplitude of the local electric field at the gaps is about 300 times larger than that of the incident field. Because of the strong enhancement of the local electric field, an electromagnetic wave with relatively low power can generate a discharge microplasma at the gap of the cutwire resonator with higher (lower) resonance frequency when the incident frequency is larger (smaller) than $f_{0}$. After the generation of a microplasma at either of the gaps, the electric field enhancement factor at the other gap drops to one eleventh of its original value, because the microplasma itself behaves as a lossy dielectric and changes the characteristics of the metasurface. Although microplasmas could be generated at both gaps simultaneously by using higher incident power, high-powered electromagnetic waves were not used in study and a microplasma was generated only at one gap.

We consider a condition in which the EIT-like metasurface behaves as a nonlinear medium. Harmonic generation was not previously observed on the EIT-like metasurface with a microplasma, as described in our previous paper 32 This implies that the metasurface with a 


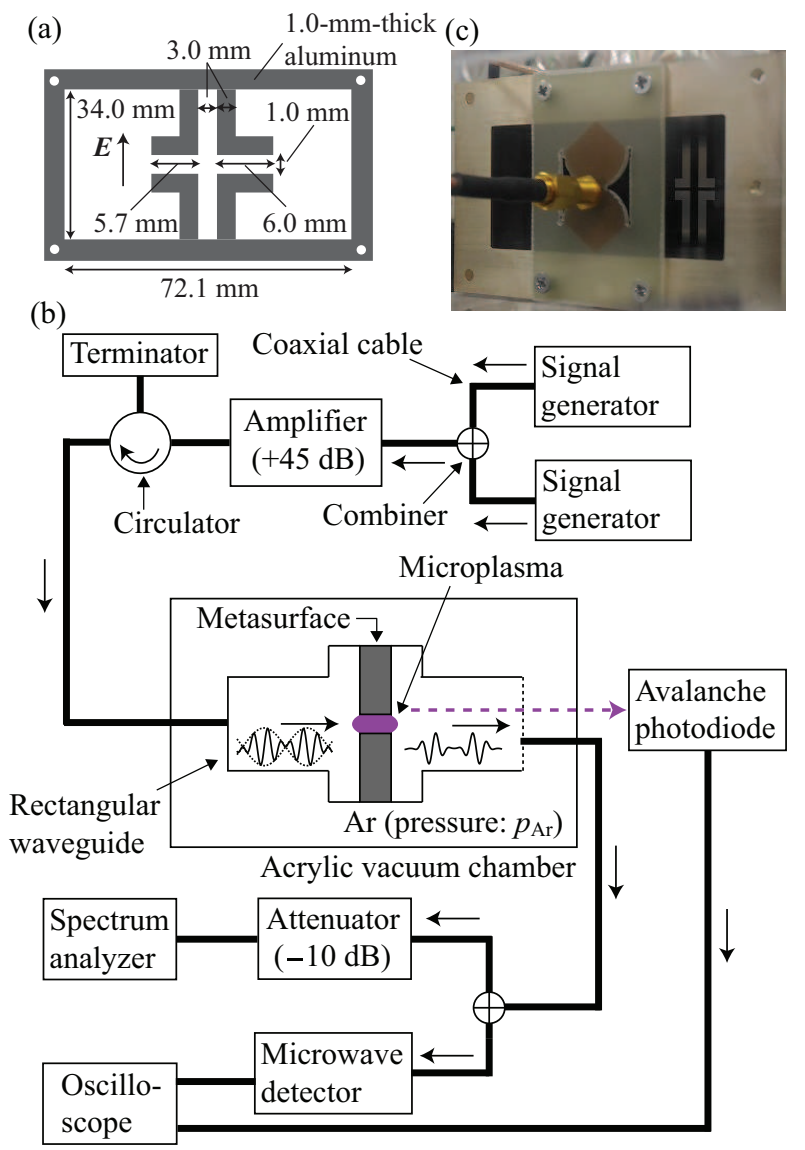

FIG. 1. (a) Schematic of the structure of the EIT-like metasurface and (b) experimental setup. The metasurface structure was combined with the flange of a rectangular waveguide to place the metasurface at the center of the waveguide. Note that the walls of the waveguide acted almost like periodic boundaries in this experiment. (c) Photograph of output end of the waveguide with antenna. Microplasma emission can be observed through the triangular apertures in the antenna substrate.

microplasma behaves as a linear medium for continuous waves. The electromagnetic response of the metasurface must be dependent on the incident field to induce a nonlinear phenomenon. That is, the generation and quenching of the microplasma needs to be dependent on the incident field. Therefore, we hypothesize that the metasurface would behave as a nonlinear medium when exposed to electromagnetic waves with temporally varying amplitudes.

We measured the transmission characteristics of the metasurface for electromagnetic waves with sinusoidally modulated amplitudes to investigate the nonlinear response of the metasurface. Figure1(b) shows a schematic of the experimental setup in this study. The metasurface was placed in a rectangular waveguide with crosssectional dimensions of $34.0 \mathrm{~mm} \times 72.1 \mathrm{~mm}$. The waveguide was placed in an acrylic vacuum chamber, where argon gas at a pressure of $p_{\mathrm{Ar}}$ was present. Two continuous
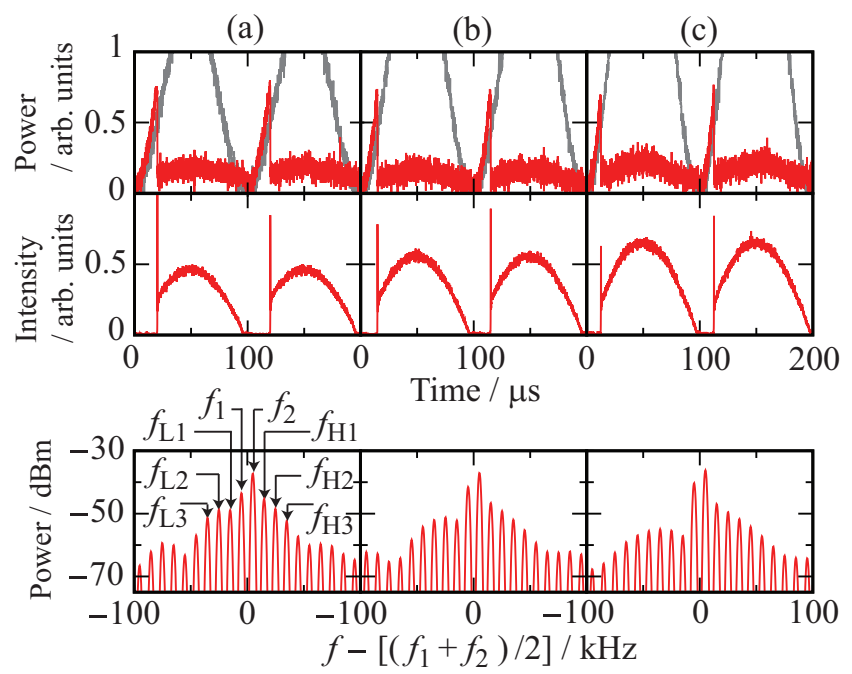

FIG. 2. The envelope of the transmitted wave (upper row), the light intensity of the microplasma emission (middle row), and the spectrum of the transmitted wave (lower row) at $p_{\text {Ar }}=0.8 \mathrm{kPa}$ for (a) $P_{0}=29.1 \mathrm{dBm}$, (b) $30.6 \mathrm{dBm}$, and (c) $32.1 \mathrm{dBm}$. The light gray curve in the upper panel shows the envelope of the transmitted wave when atmospheric-pressure air filled the chamber and a microplasma was not generated at the metasurface.

waves with the same power were generated by two signal generators, and these waves were combined to generate a sinusoidally modulated incident wave. The frequencies of these two continuous waves were $f_{1}=f_{0}=3.031 \mathrm{GHz}$ and $f_{2}=f_{1}+10 \mathrm{kHz}$. For this frequency condition, a microplasma was generated at the gap of the cut-wire resonator with the higher resonance frequency, which is at the left side of Fig.11(a). The sinusoidally modulated wave was amplified using an amplifier. The power of each continuous wave after amplification was defined as $P_{0}$. The amplified wave was introduced into the waveguide through a coaxial-to-waveguide transformer, and was incident on the metasurface. The transmitted wave was received by an ultra-wideband dipole antenna. $\stackrel{37}{\text { The re- }}$ ceived wave was split into two halves. One half was detected by a microwave diode detector to observe the envelope of the transmitted wave using an oscilloscope. The other half was fed into a spectrum analyzer. The microplasma emission at the gap of the cut-wire resonator with the higher resonance frequency was observed through an aperture fabricated in the receiving antenna substrate, as shown in Fig.1(c), and detected by an avalanche photodiode. The output signal of the photodiode was fed into the oscilloscope. We measured the envelope of the transmitted wave and the light intensity of the microplasma emission, as well as the spectrum of the transmitted wave as $P_{0}$ and $p_{\text {Ar }}$ were varied.

Figure 2 shows examples of the measured characteristics of the transmitted wave and microplasma emission. The upper and middle panels show the envelope of the 
(a)

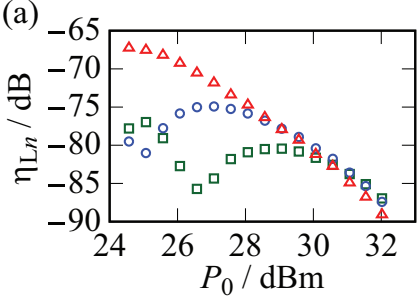

(b)

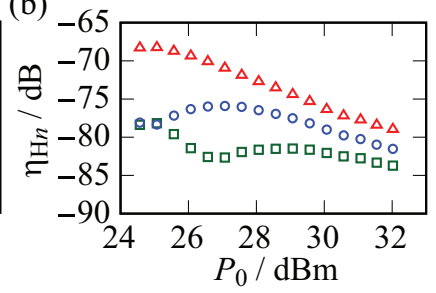

FIG. 3. Incident power dependence of frequency conversion efficiency at $p_{\mathrm{Ar}}=0.8 \mathrm{kPa}$ for (a) lower sidebands and (b) upper sidebands. Triangles, circles, and squares correspond to $n=1,2$, and 3 , respectively.

transmitted wave and the intensity of the microplasma emission, respectively. As the incident amplitude increases from zero, the transmission amplitude first increases and then drops sharply. Further increases and subsequent decreases in the incident amplitude cause little change in the transmission amplitude. After the incident amplitude becomes smaller than a certain value, the transmission amplitude varies with the incident amplitude again. The light intensity of the microplasma emission varies periodically, with a period equal to the modulation period of the incident amplitude. The duration of the low-transmittance state agrees with the time for microplasma emission. Thus, the temporal variation of the transmittance is confirmed to be caused by the generation and quenching of the microplasma. The result that the transmittance becomes low during microplasma generation agrees with our previous results $\stackrel{32,33}{ }$ As $P_{0}$ increases, the ratio of the duration of microplasma generation to the modulation period increases. This is because the duration that the incident amplitude exceeds the threshold value for plasma ignition increases with $P_{0}$. The lower panel of Fig.2 2 shows the spectrum of the transmitted wave. Many sidebands are observed in the spectrum and the power of each sideband varies with $P_{0}$. The frequency separation between the sidebands is $10 \mathrm{kHz}$, which equals $f_{2}-f_{1}$. These results in both the time and frequency domains clarify that frequency mixing can be realized using the generation and quenching of a microplasma on the EIT-like metasurface.

Next, we investigate the frequency mixing phenomenon in detail. Figure 3 shows the incident power dependence of the frequency conversion efficiency. Here, we define the frequency conversion efficiency as $\eta_{\{\mathrm{L}, \mathrm{H}\} n}=P_{\{\mathrm{L}, \mathrm{H}\}_{n}} / P_{0}$ $(n=1,2,3, \cdots)$, where $P_{\{\mathrm{L}, \mathrm{H}\} n}$ is the power of a sideband with frequency $f_{\{\mathrm{L}, \mathrm{H}\} n}$, as indicated in Fig.22, The conversion efficiency is increased or decreased by increasing the incident power, depending on $P_{0}$ and $n$. This observation is quite different compared with typical nonlinear phenomena, in which frequency conversion efficiency always increases with incident power.

Let us consider a simple model of the EIT-like metasurface to understand the dependence of the frequency conversion efficiency on $P_{0}$. The transmittance of the (a)
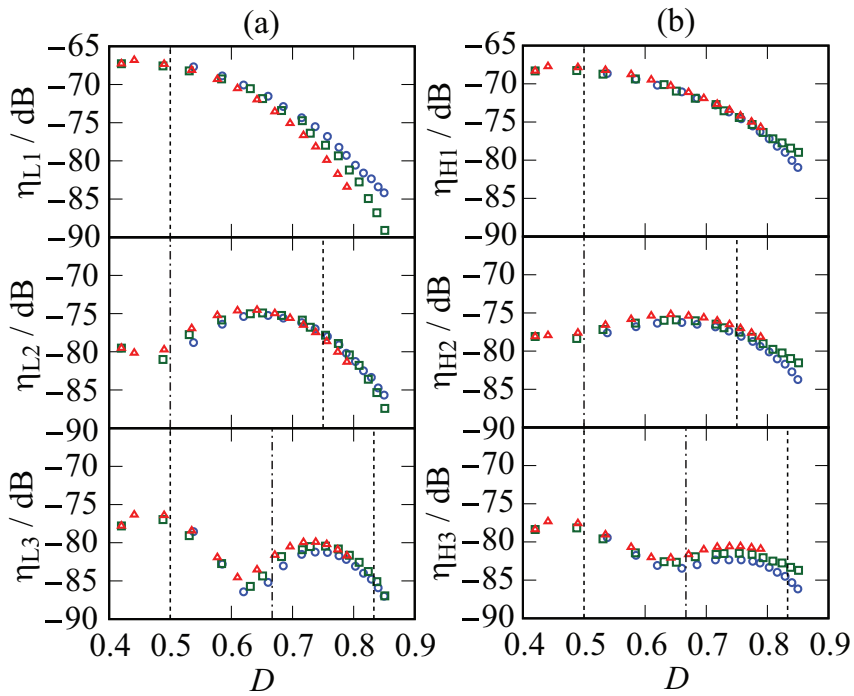

FIG. 4. Dependence of frequency conversion efficiency for (a) lower sidebands and (b) upper sidebands on the ratio of plasma generation duration to modulation period. Triangles, squares, and circles correspond to experimental data at $p_{\mathrm{Ar}}=$ $0.4 \mathrm{kPa}, 0.8 \mathrm{kPa}$, and $1.2 \mathrm{kPa}$, respectively. Vertical dashed lines and dot-and-dash lines represent values of $D$ for which $\left|c_{n}\right|$ becomes a local maximum or minimum, respectively.

metasurface with a microplasma is relatively low, and nearly independent of the incident amplitude. Therefore, we assume that the transmittance of the metasurface only depends on whether the microplasma is generated or not. That is, the amplitude transmittances with and without the microplasma are assumed to be $a_{1}$ and $a_{2}\left(a_{2}>a_{1}\right)$, respectively. The time dependence of the amplitude transmittance $a(t)$ is a periodic function with a period of $T=100 \mu \mathrm{s}=\left(f_{2}-f_{1}\right)^{-1}$ in this experiment. Thus, $a(t)$ can be expanded into Fourier series as follows: $a(t)=D a_{1}+(1-D) a_{2}+$ $\sum_{n=1}^{\infty}\left[c_{n} \exp (-\mathrm{i} 2 n \pi t / T)+c_{n}^{*} \exp (\mathrm{i} 2 n \pi t / T)\right]$, where $D$ is the ratio of the duration of microplasma generation to $T,\left|c_{n}\right|=\left[\left(a_{2}-a_{1}\right) /(n \pi)\right]|\sin [n \pi(1-D)]|$, and * indicates the complex conjugate. The time dependence of the transmitted amplitude is described by the product of the incident amplitude and $a(t)$. Therefore, the spectrum of the transmitted wave can be expressed as the convolution of the Fourier transforms of the incident wave and $a(t)$. Assuming that the lower (upper) sidebands can be expressed as the convolution of the Fourier transforms of a continuous wave with frequency of $f_{1}\left(f_{2}\right)$ and $a(t)$ for simplicity, the powers of the sidebands are described by $\left|c_{n}\right|$, where the only parameter that depends on the experimental condition is $D$.

To compare this model with the experiment, we show in Fig. 4 the dependence of $\eta_{\{\mathrm{L}, \mathrm{H}\} n}(n=1,2,3)$ on $D$ that was measured for various values of $P_{0}(\leq 32.1 \mathrm{dBm})$ at $p_{\mathrm{Ar}}=0.4 \mathrm{kPa}, 0.8 \mathrm{kPa}$, and $1.2 \mathrm{kPa}$. Here, $D$ was 
determined from the light intensity of the microplasma emission. The conversion efficiencies are found to be determined almost entirely by $D$, even though $P_{0}$ and $p_{\text {Ar }}$ are tested at various values. In addition, the values of $D$ for which $\eta_{\{\mathrm{L}, \mathrm{H}\} n}$ becomes local maxima or minima in the experiment roughly agree with those calculated from the model. This implies that the frequency mixing is derived from the binary change in the transmittance, which is caused by the generation and quenching of the microplasma. The discrepancy between the experiment and model in $D$ for which $\eta_{\{\mathrm{L}, \mathrm{H}\} n}$ becomes a local maximum or minimum is relatively large in the region of larger $D$ values, which may be caused by oversimplifications in the model.

The nonlinear response in the present experiment cannot be expressed as a $n$-th order nonlinearity. In typical nonlinear media, the ratio of the polarization density to the electric field depends on the electric field. However, the ratio in the metasurface depends instead on the amplitude of the electric field. In this way, the nonlinear response at this metasurface is similar to that of a rf-SQUID metamaterial $\stackrel{15}{\underline{n}}$ The abrupt change in the electromagnetic response observed in these metamaterials may be useful for the efficient generation of various nonlinear phenomena.

In conclusion, we investigated the nonlinear response of an EIT-like metasurface loaded with gas as a nonlinear element to develop a method for preventing the suppression of the enhancement of the local electromagnetic field in a resonant metamaterial. We measured the transmission characteristics of the metasurface and the light intensity of the microplasma emission for an incident wave with a sinusoidally modulated amplitude to verify the generation of nonlinear phenomenon. The measured envelope and spectrum of the transmitted wave, as well as the temporal dependence of the microplasma emission, revealed that frequency mixing occurred at the metasurface because of the generation and quenching of the microplasma. The parameter dependence of the frequency conversion efficiency showed that the efficiency was determined almost entirely by the ratio of duration of the microplasma generation to the modulation period. The metasurface may be classified into a kind of waveform selective metasurface $\frac{38,39}{}$ because the time variation of the incident amplitude is essential for the generation of nonlinear phenomenon. Note that the nonlinear phenomenon in this study is not caused by a nonlinearity in the plasma, in contrast with some previous works $\underline{40} \underline{-42}$ Although harmonic generation has not been observed in this metasurface with microplasma, it can be generated in principle and should be investigated in a future study. It should be added that the interactions between free electrons in conductors and the enhanced local magnetic field can be used as another way to prevent the suppression of the enhancement of the local electromagnetic field for efficient generation of nonlinear phenomena. ${ }^{43}$

This research was supported in part by JSPS KAKENHI Grant Number JP16K14249, and by a re- search grant from The Murata Science Foundation.

${ }^{1}$ M. Lapine, I. V. Shadrivov, and Y. S. Kivshar, Rev. Mod. Phys. 86, 1093 (2014).

${ }^{2}$ T. Kanazawa, Y. Tamayama, T. Nakanishi, and M. Kitano, Appl. Phys. Lett. 99, 024101 (2011).

${ }^{3}$ T. Nakanishi, Y. Tamayama, and M. Kitano, Appl. Phys. Lett. 100, 044103 (2012).

${ }^{4}$ R. Czaplicki, H. Husu, R. Siikanen, J. Mäkitalo, M. Kauranen, J. Laukkanen, J. Lehtolahti, and M. Kuittinen, Phys. Rev. Lett. 110, 093902 (2013).

${ }^{5}$ M. Celebrano, X. Wu, M. Baselli, S. Großmann, P. Biagioni, A. Locatelli, C. D. Angelis, G. Cerullo, R. Osellame, B. Hecht, F. C. L. Duò, and M. Finazzi, Nature Nano. 10, 412 (2015).

${ }^{6}$ K. O'Brien, H. Suchowski, J. Rho, A. Salandrino, B. Kante, X. Yin, and X. Zhang, Nature Mater. 14, 379 (2015).

${ }^{7}$ Y. Yang, W. Wang, A. Boulesbaa, I. I. Kravchenko, D. P. Briggs, A. Puretzky, D. Geohegan, and J. Valentine, Nano Lett. 15, 7388 (2015).

${ }^{8}$ S. Liu, M. B. Sinclair, S. Saravi, G. A. Keeler, Y. Yang, J. Reno, G. M. Peake, F. Setzpfandt, I. Staude, T. Pertsch, and I. Brener, Nano Lett. 16, 5426 (2016).

${ }^{9}$ A. S. Shorokhov, E. V. Melik-Gaykazyan, D. A. Smirnova, B. Hopkins, K. E. Chong, D.-Y. Choi, M. R. Shcherbakov, A. E. Miroshnichenko, D. N. Neshev, A. A. Fedyanin, and Y. S. Kivshar, Nano Lett. 16, 4857 (2016).

${ }^{10} \mathrm{~J}$. Alberti, H. Linnenbank, S. Linden, Y. Grynko, and J. Förstner, Appl. Phys. B 122, 45 (2016).

${ }^{11}$ D. Filonov, Y. Kramer, V. Kozlov, B. A. Malomed, and P. Ginzburg, Appl. Phys. Lett. 109, 111904 (2016).

${ }^{12}$ S. D. Gennaro, M. Rahmani, V. Giannini, H. Aouani, T. P. H. Sidiropoulos, M. Navarro-Cía, S. A. Maier, and R. F. Oulton, Nano Lett. 16, 5278 (2016).

${ }^{13}$ B. Metzger, M. Hentschel, M. Nesterov, T. Schumacher, M. Lippitz, and H. Giessen, Appl. Phys. B 122, 77 (2016).

${ }^{14} \mathrm{H}$. Su, Y. Guo, W. Gao, J. Ma, Y. Zhong, W. Y. Tam, C. T. Chan, and K. S. Wong, Sci. Rep. 6, 22061 (2016).

${ }^{15}$ D. Zhang, M. Trepanier, T. Antonsen, E. Ott, and S. M. Anlage, Phys. Rev. B 94, 174507 (2016).

${ }^{16}$ F. Wang, A. B. F. Martinson, and H. Harutyunyan, ACS Photon. 4, 1188 (2017).

${ }^{17}$ O. Wolf, S. Campione, Y. Yang, and I. Brener, Sci. Rep. 7, 8101 (2017).

${ }^{18}$ K.-Y. Yang, J. Butet, C. Yan, G. D. Bernasconi, and O. J. F. Martin, ACS Photon. 4, 15221530 (2017).

${ }^{19}$ M. Liu, H. Y. Hwang, H. Tao, A. C. Strikwerda, K. Fan, G. R. Keiser, A. J. Sternbach, K. G. West, S. Kittiwatanakul, J. Lu, S. A. Wolf, F. G. Omenetto, X. Zhang, K. A. Nelson, and R. D. Averitt, Nature 487, 345 (2012).

${ }^{20}$ M. Ren, E. Plum, J. Xu, and N. I. Zheludev, Nature Commun. 3, 833 (2012).

${ }^{21}$ K. Fan, H. Y. Hwang, M. Liu, A. C. Strikwerda, A. Sternbach, J. Zhang, X. Zhao, X. Zhang, K. A. Nelson, and R. D. Averitt, Phys. Rev. Lett. 110, 217404 (2013).

${ }^{22}$ S. Guddala and S. A. Ramakrishna, Opt. Lett. 41, 5150 (2016).

${ }^{23}$ V. Savinov, K. Delfanazari, V. A. Fedotov, and N. I. Zheludev, Appl. Phys. Lett. 108, 101107 (2016).

${ }^{24}$ G. R. Keiser, N. Karl, P. Q. Liu, C. Tulloss, H.-T. Chen, A. J. Taylor, I. Brener, J. L. Reno, and D. M. Mittleman, Appl. Phys. Lett. 111, 121101 (2017).

${ }^{25}$ M. Lawrence, I. D. R. Barton, and J. A. Dionne, Nano Lett. 18, 1104 (2018).

${ }^{26}$ I. V. Shadrivov, A. A. Zharov, N. A. Zharova, and Y. S. Kivshar, Photon. Nanostr. Fundam. Appl. 4, 69 (2006).

${ }^{27}$ R. E. Noskov, P. A. Belov, and Y. S. Kivshar, Phys. Rev. Lett. 108, 093901 (2012).

${ }^{28}$ Y. Tamayama, T. Nakanishi, and M. Kitano, Phys. Rev. B 87, 195123 (2013).

${ }^{29}$ A. B. Kozyrev, I. V. Shadrivov, and Y. S. Kivshar, Appl. Phys. Lett. 104, 084105 (2014). 
${ }^{30}$ M. Agaoglou, V. M. Rothos, D. J. Frantzeskakis, G. P. Veldes, and H. Susanto, Int. J. Bifurcat. Chaos 24, 1450147 (2014).

${ }^{31}$ B. Rai and A. R. McGurn, Phys. Rev. B 91, 085113 (2015).

${ }^{32}$ Y. Tamayama, K. Hamada, and K. Yasui, Phys. Rev. B 92, 125124 (2015).

${ }^{33}$ Y. Tamayama and O. Sakai, J. Appl. Phys. 121, 073303 (2017).

${ }^{34}$ M. Fleischhauer, A. Imamoglu, and J. P. Marangos, Rev. Mod. Phys. 77, 633 (2005).

${ }^{35}$ C. L. Garrido Alzar, M. A. G. Martinez, and P. Nussenzveig, Am. J. Phys. 70, 37 (2002).

${ }^{36}$ Y. Tamayama, K. Yasui, T. Nakanishi, and M. Kitano, Phys. Rev. B 89, 075120 (2014).
${ }^{37}$ E. Lule, T. Babi, and K. Siwiak, Microw. Opt. Technol. Lett. 46, 536 (2005).

${ }^{38}$ H. Wakatsuchi, J. Appl. Phys. 117, 164904 (2015).

${ }^{39}$ H. Wakatsuchi, F. Gao, S. Yagitani, and D. F. Sievenpiper, Sci. Rep. 6, 31371 (2016).

${ }^{40}$ A. Iwai, Y. Nakamura, and O. Sakai, Phys. Rev. E 92, 033105 (2015).

${ }^{41}$ A. Iwai, Y. Nakamura, A. Bambina, and O. Sakai, Appl. Phys. Express 8, 056201 (2015).

${ }^{42}$ J. Gregório, S. Parsons, and J. Hopwood, Plasma Sources Sci. Technol. 25, 035018 (2016).

${ }^{43}$ Y. Wen and J. Zhou, Phys. Rev. Lett. 118, 167401 (2017). 